\title{
Costs of Disease Relapses Among Individuals with Granulomatosis, with Polyangiitis, or Microscopic Polyangiitis in the United States
}

\author{
Amanda M. Kong · Gilwan Kim · Margaret Michalska · Jennie H. Best
}

Received: November 14, 2017 / Published online: February 22, 2018

(C) The Author(s) 2018. This article is an open access publication

\begin{abstract}
Introduction: Granulomatosis with polyangiitis (GPA) and microscopic polyangiitis (MPA) are two related forms of systemic vasculitis. Patients with these conditions often experience relapses affecting various body systems. Here we describe rates of relapse and review healthcare costs resulting from relapse among patients with GPA/MPA.

Methods: Two groups of patients with GPA and MPA were selected from the MarketScan claims databases between 2011 and 2013 based on diagnosis codes. Patients were followed for 12 months to identify relapses based on an algorithm of diagnoses in medical and medication claims. Relapses were categorized into one of the following groups: renal relapse, pulmonary relapse, other relapse-associated
\end{abstract}

Enhanced content To view enhanced content for this article go to https://doi.org/10.6084/m9.figshare. 5863926.

Electronic supplementary material The online version of this article (https://doi.org/10.1007/s40744018-0099-1) contains supplementary material, which is available to authorized users.

A. M. Kong ( $₫)$ · G. Kim

Truven Health Analytics, an IBM Company,

Cambridge, MA, USA

e-mail: akong@us.ibm.com

M. Michalska · J. H. Best

Genentech, Inc, South San Francisco, CA, USA condition relapse, GPA or MPA utilization relapse, and mixed relapse.

Results: The final sample of patients with GPA and MPA consisted of 2707 and 740 patients, respectively. In both groups, approximately one-quarter of patients experienced relapse during the 12-month follow-up period. The mean all-cause healthcare costs in the 4-month period after relapse were $\$ 38,313(\mathrm{SD}, \$ 54,120$ ) for patients with GPA and $\$ 35,947$ (SD, $\$ 48,065)$ for patients with MPA. In both groups, renal relapses were the costliest. Costs during the 4 months immediately following relapses were substantially higher than what could be expected over a 4-month follow-up among patients who did not experience relapse based on 12-month all-cause costs (GPA, \$32,005 [SD, \$64,570]; MPA, \$61,044 [SD, \$125,093]).

Conclusions: Relapses are common among patients with GPA and MPA, and treatment of relapses can be costly. More effective therapies are needed to prevent relapses.

Funding: Genentech, Inc.

Keywords: Autoimmune disease;

Granulomatosis with polyangiitis; Health care costs; Relapse

\section{INTRODUCTION}

Granulomatosis with polyangiitis (GPA), formerly known as Wegener's granulomatosis, and 
microscopic polyangiitis (MPA) are two types of antineutrophil cytoplasmic autoantibody (ANCA)-associated vasculitis [1]. Both conditions are characterized by inflammation of small- and medium-sized arteries [1]. Inflammation of small blood vessels can lead to tissue damage, typically in the respiratory tract and the kidneys [1]. In GPA, granulomas, characteristic conglomerates of necrotic tissue and inflammatory cells, can be present. Granulomas are not typically identified in MPA [1]. Current treatment options include cytotoxic agents (such as cyclophosphamide and methotrexate) or a monoclonal antibody (rituximab), in combination with corticosteroids [2-4].

Despite treatment, disease recurrences are very common. Studies have estimated that that 30 to greater than $50 \%$ of patients with GPA experience a relapse within a 4 -year period following the initial diagnosis [5-8]. In clinical practice, GPA relapses are generally identified using the Birmingham Vasculitis Activity Scale (BVAS) [9]. The scale includes several conditions such as cutaneous ulcers, limb gangrene, mesenteric ischemia, respiratory failure, stroke, and decrease in kidney function [9]. Treating these events, including with medications and procedures, such as renal dialysis, can be very costly. A previously conducted analysis, which used US administrative healthcare claims data, found that during a 1-year follow-up, patients with GPA, who experienced a major relapse, incurred healthcare costs that were more than double the costs of patients who did not experience a relapse [10]. However, that analysis did not evaluate relapses among patients with MPA and did not attempt to classify relapses by the organ system. Therefore, the aim of this analysis was to describe the burden of illness of relapse for patients with GPA or MPA by type of relapse. Specifically, the objectives of the current analysis were to: (1) identify the rates of major relapse among patients with MPA, (2) identify the rates of pulmonary and renal relapses in patients with GPA and MPA, and (3) to assess the healthcare costs incurred following a major relapse.

\section{METHODS}

\section{Data Source}

This analysis was conducted in the Truven Health MarketScan Commercial and Medicare Supplemental administrative claims databases. The data include enrollment records, inpatient and outpatient medical claims, and outpatient pharmacy claims linked by a unique enrollee identifier. Enrollees include individuals insured through a variety of health insurance plans, including fee-for-service and capitated plans. These databases have been utilized in many previously published analyses [11]. The data were previously collected, statistically de-identified, and HIPAA compliant; therefore, ethics approval for this study was not required in accordance with the policies of Truven Health Analytics. This article is based on previously conducted data, and does not involve any new studies of human or animal subjects performed by any of the authors.

\section{Patient Selection}

Patients with $\geq 2$ non-diagnostic claims (i.e., claims that did not have procedure codes for laboratory or radiology tests) with a diagnosis of GPA (ICD-9-CM 446.4) in any position during the period from March 1, 2011, to March 1, 2013 , were selected. The date of the first claim with a GPA diagnosis during that period was designated as the index date. Patients must have been $\geq 18$ years old and continuously enrolled for $\geq 12$ months following the index date. Patients who had continuous enrollment for $\geq 12$ months prior to the index date with no claims with a GPA diagnosis during that time (i.e., a 12-month clean period) were classified as incident patients with GPA. All other patients were classified as prevalent patients with GPA.

There is no specific ICD-9-CM diagnosis code for MPA. Therefore, to select patients with MPA, all patients with $\geq 2$ non-diagnostic claims coded as unspecified arteritis (ICD-9-CM 447.6), between March 1, 2011 and March 1, 2013, were identified. Again, the date of the earliest claim of the unspecified arteritis diagnosis 
during the 2-year period was designated as the index date. Patients were required to be $\geq 18$ years old and continuously enrolled for $\geq 12$ months following the index date. In order to fulfill additional criteria, all potential patients with MPA were also required to have been enrolled for 12 months prior to the index date. Patients were required to have had $\geq 1$ non-diagnostic claim with a diagnosis of acute or chronic renal failure, glomerulonephritis, or hemoptysis during 1 month prior to or 6 months after the index date, as these conditions are recognized as manifestations of MPA. Lastly, patients who had $\geq 1$ non-diagnostic claim with a diagnosis of GPA, hepatitis B, or hepatitis $C$ during the 12 months prior to the index date and up to 24 months after the index date were excluded, to avoid misclassification of MPA. Patients with MPA who had no claims with the unspecified arteritis diagnosis during the 12 months before the index date were considered incident cases; all others were considered prevalent cases. Patients with GPA or MPA were selected from the MarketScan databases using previously published algorithms [10].

\section{Study Design}

This study was a retrospective analysis. As described above, the index date was the date of first diagnosis of GPA or MPA in the claims data between March 1, 2011, and March 1, 2013. The follow-up period was defined as the 12 months after the index date. The presence of relapse was evaluated during the 12-month follow-up period. Costs were measured both during the 4 months following a relapse, based on the average duration of relapses from the RAVE trial, and over the 12-month follow-up period after the index date [12].

\section{Variables}

Demographic characteristics were established on the index date using enrollment records and clinical characteristics were assessed in the follow-up period for all patients using medical claims. Demographic characteristics included age, sex, and geographic region. Clinical characteristics captured included the Deyo-Charlson Comorbidity Index (CCI) [13] and the diagnoses of relapse-associated conditions, based on the major items in the BVAS scale, listed in any position in a non-diagnostic claim (acute/chronic renal failure, gangrene, scleritis, retinal exudates/hemorrhage, sensorineural deafness, mesenteric ischemia, alveolar hemorrhage, respiratory failure, meningitis, spinal cord lesion, stroke, cranial nerve palsy, sensory peripheral neuropathy, motor mononeuritis multiplex). It should be noted that no ICD-9-CM diagnosis code exists that is specific to alveolar hemorrhage; therefore, the code for hemoptysis was used. Diagnosis codes for relapse-associated conditions can be found in Supplemental Table S1.

The presence of a major relapse in the followup period was determined by an algorithm based on diagnoses in medical claims and medication use [10]. For incident patients, claims in the first 30 days after the index date were not evaluated for relapse, in order to avoid counting the initial diagnosis as a relapse event. The first step in the algorithm was to identify a non-diagnostic inpatient or emergency room claim with the diagnosis of GPA (or unspecified arteritis diagnosis for MPA patients) in any position, $\geq 2$ non-diagnostic outpatient office visit claims coded as GPA (or unspecified arteritis diagnosis for patients with MPA) in any position in a 30-day period, or a non-diagnostic claim with a diagnosis of a relapse-associated condition listed. If present, the earliest date that met these criteria was considered a potential relapse date. Following the identification of one of these three criteria, the claims available within the next 30 days were screened for the presence of a claim for a medication commonly used in active therapy (oral or intravenous cyclophosphamide, rituximab, oral corticosteroids with prednisone-equivalent dose of $>20 \mathrm{mg}$, or intravenous corticosteroids). Finally, if a claim for one of the aforementioned medications was present and the patient had no evidence of a relapse in the 30 days prior to the potential relapse date (no claims for medications or claims meeting the criteria considered in the first step of the algorithm were identified), the event was classified as a major relapse, 
and the potential relapse date was designated as the relapse date. This algorithm, which was designed to identify major relapse in patients with GPA using claims data, has been previously published [10]. Relapses of GPA and MPA during the 12-month follow-up period were captured.

Each relapse during the follow-up period was classified into one of five types (renal relapse, pulmonary relapse, other relapse-associated-condition relapse, GPA [or MPA] utilization relapse, and mixed relapse) using the claims on the relapse date. If there was a claim with a diagnosis of acute or chronic renal failure on the relapse date, the relapse was considered to be a renal relapse. Similarly, if there was a diagnosis of hemoptysis or respiratory failure, the relapse was considered to be a pulmonary relapse. If a diagnosis of another relapse-associated condition based on the major items of the BVAS was recorded, the relapse was categorized as an "other relapse-associated condition" relapse. Finally, if these diagnoses were not present but the relapse was identified based on an inpatient admission or an emergency room claim with a GPA (or unspecified arteritis) diagnosis, or two outpatient office visit claims with a GPA (or unspecified arteritis) diagnosis within a 30-day window, the relapse was considered a utilization relapse. A relapse that met the definition for $>1$ category was considered a mixed relapse.

To estimate the cost of a relapse, the amount paid (including insurer-paid and patient-paid portions) on the inpatient and outpatient medical claims, as well as outpatient pharmacy claims in the 4 months after the first relapse date, were captured. Both all-cause costs (regardless of the diagnosis on the claims) and disease-specific costs (i.e., GPA-specific costs and MPA-specific costs) were measured. Diseasespecific costs were the sum of the amount paid on medical claims with the GPA diagnosis in any position or unspecified arteritis diagnosis in any position for patients with MPA, medical claims with a diagnosis of a relapse-associated condition in any position, or an outpatient pharmacy claim for GPA and MPA pharmacotherapies (corticosteroids, cyclophosphamide, methotrexate, azathioprine, leflunomide, rituximab, or mycophenolate mofetil). All-cause and disease-specific costs were measured for the following service categories: inpatient stay, emergency department visit, outpatient office visit, other outpatient services, and outpatient pharmacy. The sum of these costs was considered the total cost. Costs were not limited to costs for caring for the specific type of relapse. For example, while allcause and GPA-specific costs after a renal relapse were captured, renal-specific costs are not presented separately. They would be included as a part of all-cause and GPA-related costs based on the aforementioned definitions of those measures. The exception was dialysis, which is commonly used for patients with renal involvement in vasculitides; thus, the inpatient and outpatient dialysis costs were specifically captured. All-cause healthcare costs and diseasespecific costs were calculated only for patients who had continuous enrollment for 4 months following the relapse. The costs following mixed relapses were included in costs after any relapse but were not reported separately. Costs were adjusted to 2014 US dollars. Healthcare costs for patients with and without relapse were also measured over the full 12-month follow-up period after the index date for comparison.

\section{Statistical Analyses}

As this research was exploratory, this analysis was descriptive in nature. Continuous variables are presented with means, standard deviations, and medians. Categorical variables are presented with counts and proportions. No statistical analyses were conducted. Analysis was conducted with SAS 9.3 (Cary, NC, USA).

\section{RESULTS}

\section{GPA Patients}

From March 1, 2011, through March 1, 2013, there were 5118 patients with $\geq 2$ non-diagnostic claims with a GPA diagnosis. After applying the study inclusion criteria, the final sample was 2707 patients, including 772 
Table 1 Demographic and clinical characteristics of patients with GPA during 12-month follow-up

\begin{tabular}{|c|c|c|c|c|c|c|}
\hline \multirow[b]{2}{*}{ Mean (SD) age } & \multicolumn{2}{|c|}{$\begin{array}{l}\text { All GPA patients } \\
(N=2707)\end{array}$} & \multicolumn{2}{|c|}{$\begin{array}{l}\text { GPA patients with } \\
\text { relapse }(N=669)\end{array}$} & \multicolumn{2}{|c|}{$\begin{array}{l}\text { GPA patients without } \\
\text { relapse }(N=2038)\end{array}$} \\
\hline & 55.6 & 15.3 & 55.1 & 15.1 & 55.7 & 15.3 \\
\hline \multicolumn{7}{|l|}{ Age group, $N(\%)$} \\
\hline $18-34$ & 284 & $10.5 \%$ & 72 & $10.8 \%$ & 212 & $10.4 \%$ \\
\hline $35-44$ & 333 & $12.3 \%$ & 87 & $13.0 \%$ & 246 & $12.1 \%$ \\
\hline $45-54$ & 524 & $19.4 \%$ & 125 & $18.7 \%$ & 399 & $19.6 \%$ \\
\hline $55-65$ & 857 & $31.7 \%$ & 220 & $32.9 \%$ & 637 & $31.3 \%$ \\
\hline $65+$ & 709 & $26.2 \%$ & 165 & $24.7 \%$ & 544 & $26.7 \%$ \\
\hline Male, $N(\%)$ & 1221 & $45.1 \%$ & 310 & $46.3 \%$ & 911 & $44.7 \%$ \\
\hline \multicolumn{7}{|l|}{ Geographic region, $N(\%)$} \\
\hline Northeast & 614 & $22.7 \%$ & 132 & $19.7 \%$ & 482 & $23.7 \%$ \\
\hline North central & 717 & $26.5 \%$ & 163 & $24.4 \%$ & 554 & $27.2 \%$ \\
\hline South & 829 & $30.6 \%$ & 214 & $32.0 \%$ & 615 & $30.2 \%$ \\
\hline West & 515 & $19.0 \%$ & 145 & $21.7 \%$ & 370 & $18.2 \%$ \\
\hline Unknown & 32 & $1.2 \%$ & 15 & $2.2 \%$ & 17 & $0.8 \%$ \\
\hline Mean (SD) Deyo CCI & 1.6 & 1.9 & 2.3 & 2.1 & 1.4 & 1.8 \\
\hline \multicolumn{7}{|c|}{ Diagnoses of major relapse-associated conditions based on BVAS*, $N(\%)$} \\
\hline Acute/chronic renal failure & 867 & $32.0 \%$ & 329 & $49.2 \%$ & 538 & $26.4 \%$ \\
\hline Gangrene & 6 & $0.2 \%$ & 4 & $0.6 \%$ & 2 & $0.1 \%$ \\
\hline Scleritis & 75 & $2.8 \%$ & 37 & $5.5 \%$ & 38 & $1.9 \%$ \\
\hline Retinal exudates/hemorrhage & 0 & $0.0 \%$ & 0 & $0.0 \%$ & 0 & $0.0 \%$ \\
\hline Sensorineural deafness & 193 & $7.1 \%$ & 74 & $11.1 \%$ & 119 & $5.8 \%$ \\
\hline Mesenteric ischemia & 12 & $0.4 \%$ & 5 & $0.7 \%$ & 7 & $0.3 \%$ \\
\hline \multicolumn{7}{|l|}{ Lung-related conditions } \\
\hline Hemoptysis* & 124 & $4.6 \%$ & 72 & $10.8 \%$ & 52 & $2.6 \%$ \\
\hline Respiratory failure & 168 & $6.2 \%$ & 110 & $16.4 \%$ & 58 & $2.8 \%$ \\
\hline Meningitis & 6 & $0.2 \%$ & 3 & $0.4 \%$ & 3 & $0.1 \%$ \\
\hline Spinal cord lesion & 3 & $0.1 \%$ & 0 & $0.0 \%$ & 3 & $0.1 \%$ \\
\hline Stroke & 45 & $1.7 \%$ & 22 & $3.3 \%$ & 23 & $1.1 \%$ \\
\hline Cranial nerve palsy & 9 & $0.3 \%$ & 4 & $0.6 \%$ & 5 & $0.2 \%$ \\
\hline Sensory peripheral neuropathy & 130 & $4.8 \%$ & 50 & $7.5 \%$ & 80 & $3.9 \%$ \\
\hline Motor mononeuritis multiplex & 26 & $1.0 \%$ & 15 & $2.2 \%$ & 11 & $0.5 \%$ \\
\hline
\end{tabular}


Table 1 continued

\begin{tabular}{llll}
\hline & $\begin{array}{l}\text { All GPA patients } \\
(N=2707)\end{array}$ & $\begin{array}{l}\text { GPA patients with } \\
\text { relapse }(N=669)\end{array}$ & $\begin{array}{l}\text { GPA patients without } \\
\text { relapse }(\boldsymbol{N}=2038)\end{array}$ \\
\hline Relapse & & & \\
Presence of any relapse, $N(\%)$ & 669 & $24.7 \%$ & \\
Presence of a renal relapse, $N(\%)$ & 215 & $7.9 \%$ \\
Presence of a pulmonary relapse, $N(\%)$ & 30 & $1.1 \%$ & \\
Presence of an "other" relapse**, $N(\%)$ & 68 & $2.5 \%$ \\
Presence of a utilization relapse, $N(\%)$ & 358 & $13.2 \%$ \\
Presence of a mixed relapse, $N(\%)$ & 62 & $2.3 \%$ & \\
\hline
\end{tabular}

BVAS Birmingham Vasculitis Activity Scale, CCI Charlson Comorbidity Index, GPA granulomatosis with polyangiitis, SD standard deviation

${ }^{*}$ Patients with hemoptysis (ICD-9 CM 786.30, 786.39) were captured because no diagnosis codes were available for alveolar hemorrhage

${ }^{* *}$ Other relapse-associated condition

incident patients. Nearly $60 \%$ of patients were over 55 years old, and nearly $50 \%$ were male (Table 1). The most common relapse-associated diagnosis during the follow-up was acute or chronic renal failure $(32.0 \%)$, followed by sensorineural deafness $(7.1 \%)$, respiratory failure $(6.2 \%)$, sensory peripheral neuropathy $(4.8 \%)$, and hemoptysis (4.6\%). During the 12-month follow-up, $669(24.7 \%)$ patients had $\geq 1$ relapse of any type. The most common types of relapse were GPA utilization-related and renal relapse.

Once the analysis was limited to patients with $\geq 4$ months of continuous enrollment after the first relapse, the number of patients with any relapse was reduced to 540 . The mean all-cause costs and GPA-related costs over the 4-month period were $\$ 35,947$ (SD, $\$ 48,065$ ) and $\$ 26,814$ (SD, \$43,605), respectively (Fig. 1 and Supplemental Table S2). Inpatient admissions and outpatient procedures, including renal dialysis, were the largest contributors to the cost of relapse management. When examining the dialysis costs alone, the mean dialysis cost among 72 patients who had $\geq 1$ claim for dialysis was $\$ 43,223$ (SD, \$55,125) (Supplemental Table S3). Total costs of renal relapses were substantially higher (all-cause: \$50,943 [SD, $\$ 65,517$ ]; GPA-related: $\$ 40,575$ [SD, \$63,085]) than those of other types of relapse. Over one- third of patients with renal relapses had $\geq 1$ claim for dialysis in the following 4 months (Supplemental Table S3). For patients with GPA with renal relapses, dialysis accounted for $36 \%$ of all-cause total costs. Among patients with dialysis, the average cost of dialysis alone was $\$ 51,734$ (SD, \$57,379). Over the 12-month period, the mean all-cause healthcare cost of patients with GPA who did not experience a relapse was $\$ 32,005$ (SD, $\$ 64,570$ ) compared to $\$ 88,631$ (SD, \$126,257) among patients with GPA who did experience a relapse.

\section{Patients with MPA}

Initially, 13,265 patients with diagnoses of unspecified arteritis were identified in the database. However, after applying the continuous enrollment requirement as well as the requirement for a diagnosis of renal failure, glomerulonephritis, or hemoptysis, the final sample consisted of 740 patients, including 507 incident cases, and only patients with serious disease were included. The average age of MPA patients was 62.0 years (SD 14.8 years), and nearly $40 \%$ of patients were male. As with patients with GPA, the most common relapseassociated diagnosis during follow-up was acute or chronic renal failure $(67.6 \%)$ (Table 2$)$. The 


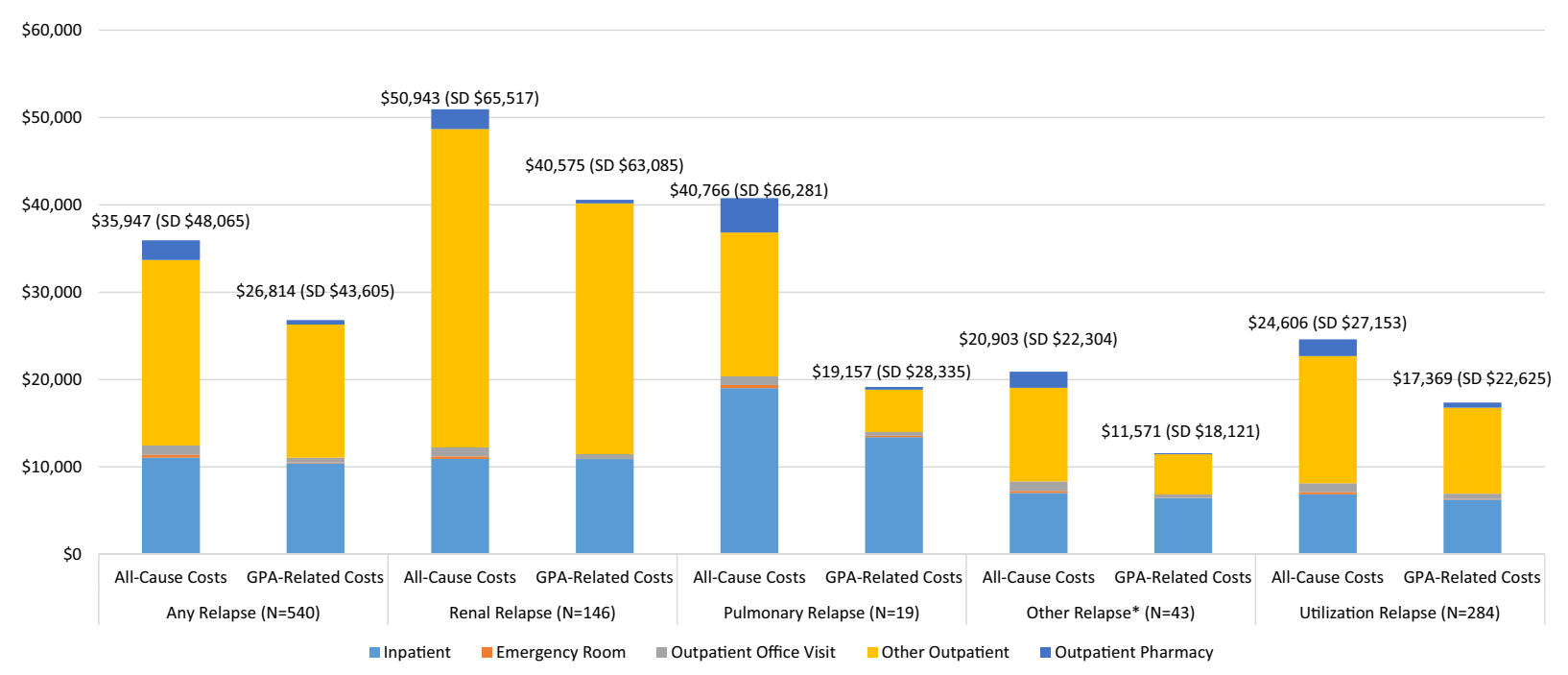

Fig. 1 Healthcare costs in the 4 months following first relapse, stratified by relapse type, for GPA patients. Note: costs were measured only among patients with 4 months of continuous enrollment following first relapse. The costs

other most-prevalent relapse-associated conditions were respiratory failure $(9.6 \%)$, sensory peripheral neuropathy (7.6\%), stroke (6.9\%), and sensorineural deafness $(6.5 \%)$. During the 12-month follow-up period, $24.3 \%$ of patients with MPA had a relapse, and $15.9 \%$ had a renal relapse (Table 2).

There were 147 patients who had a relapse and had $\geq 4$ months of continuous enrollment following their first relapse. The average total all-cause cost over the 4-month period after the first relapse was $\$ 38,313$ (SD $\$ 54,120$ ), and $59.5 \%$ of costs were classified as MPA-related (mean \$22,784; SD \$42,810) (Fig. 2 and Supplemental Table S2). The primary drivers of cost were inpatient admissions and outpatient services. Renal relapses were the most costly, with mean total all-cause costs of $\$ 42,802$ (SD $\$ 60,236)$. Approximately one-quarter $(23.4 \%)$ of patients experiencing renal relapses had $\geq 1$ claim for dialysis. For patients with MPA with a renal relapse, dialysis accounted for $18 \%$ of allcause total costs. Average dialysis cost among patients who had a renal relapse and required dialysis was \$32,143 (SD \$38,539) (Supplemental Table S3). Over the 12-month period after following mixed relapses were included in costs after any relapse but were not reported separately. GPA granulomatosis with polyangiitis. Asterisk other relapse associated condition

the index date, the mean all-cause healthcare cost of patients with MPA who did not experience a relapse was $\$ 61,044$ (SD $\$ 125,093$ ) and was $\$ 111,691$ (SD $\$ 156,189$ ) for patients with MPA who did experience a relapse.

\section{DISCUSSION}

In this claims-based analysis of individuals with GPA and MPA, disease relapses were not uncommon. Among patients with GPA, utilization relapse was the most common type of relapse, and renal relapse was the second most common. Among patients with MPA, renal relapse was the most common type of relapse. The cost of care for renal relapses was greater than that for other types of relapses, with allcause costs during the 4 months following a relapse averaging $>\$ 50,000$ in patients with GPA and $>\$ 42,000$ for patients with MPA. The major contributors to these costs were inpatient admissions and outpatient services, including renal dialysis. It is important to emphasize that the costs of services provided within 4 months after a relapse are remarkably greater than the 
Table 2 Demographic and clinical characteristics of patients with MPA during 12-month follow-up

\begin{tabular}{|c|c|c|c|c|c|c|}
\hline \multirow[b]{2}{*}{ Mean (SD) age } & \multicolumn{2}{|c|}{$\begin{array}{l}\text { All MPA patients } \\
(N=740)\end{array}$} & \multicolumn{2}{|c|}{$\begin{array}{l}\text { MPA patients with } \\
\text { relapse }(N=180)\end{array}$} & \multicolumn{2}{|c|}{$\begin{array}{l}\text { MPA patients without } \\
\text { relapse }(N=560)\end{array}$} \\
\hline & 62.0 & 14.8 & 63.4 & 15.0 & 61.6 & 14.8 \\
\hline \multicolumn{7}{|l|}{ Age group, $N(\%)$} \\
\hline $18-34$ & 34 & $4.6 \%$ & 8 & $4.4 \%$ & 26 & $4.6 \%$ \\
\hline $35-44$ & 40 & $5.4 \%$ & 12 & $6.7 \%$ & 28 & $5.0 \%$ \\
\hline $45-54$ & 140 & $18.9 \%$ & 24 & $13.3 \%$ & 116 & $20.7 \%$ \\
\hline $55-65$ & 233 & $31.5 \%$ & 56 & $31.1 \%$ & 177 & $31.6 \%$ \\
\hline $65+$ & 293 & $39.6 \%$ & 80 & $44.4 \%$ & 213 & $38.0 \%$ \\
\hline Male, $N(\%)$ & 293 & $39.6 \%$ & 62 & $34.4 \%$ & 231 & $41.3 \%$ \\
\hline \multicolumn{7}{|l|}{ Geographic region, $N(\%)$} \\
\hline Northeast & 176 & $23.8 \%$ & 47 & $26.1 \%$ & 129 & $23.0 \%$ \\
\hline North Central & 215 & $29.1 \%$ & 56 & $31.1 \%$ & 159 & $28.4 \%$ \\
\hline South & 221 & $29.9 \%$ & 47 & $26.1 \%$ & 174 & $31.1 \%$ \\
\hline West & 124 & $16.8 \%$ & 29 & $16.1 \%$ & 95 & $17.0 \%$ \\
\hline Unknown & 4 & $0.5 \%$ & 1 & $0.6 \%$ & 3 & $0.5 \%$ \\
\hline Mean (SD) Deyo CCI & 3.1 & 2.3 & 3.9 & 2.3 & 2.8 & 2.2 \\
\hline \multicolumn{7}{|c|}{ Diagnoses of major relapse-associated conditions based on BVAS,$N(\%)$} \\
\hline Acute/chronic renal failure & 500 & $67.6 \%$ & 159 & $88.3 \%$ & 341 & $60.9 \%$ \\
\hline Gangrene & 25 & $3.4 \%$ & 6 & $3.3 \%$ & 19 & $3.4 \%$ \\
\hline Scleritis & 8 & $1.1 \%$ & 2 & $1.1 \%$ & 6 & $1.1 \%$ \\
\hline Retinal exudates/hemorrhage & 0 & $0.0 \%$ & 0 & $0.0 \%$ & 0 & $0.0 \%$ \\
\hline Sensorineural deafness & 48 & $6.5 \%$ & 14 & $7.8 \%$ & 34 & $6.1 \%$ \\
\hline Mesenteric ischemia & 11 & $1.5 \%$ & 4 & $2.2 \%$ & 7 & $1.3 \%$ \\
\hline \multicolumn{7}{|l|}{ Lung-related conditions } \\
\hline Hemoptysis* & 39 & $5.3 \%$ & 12 & $6.7 \%$ & 27 & $4.8 \%$ \\
\hline Respiratory failure & 71 & $9.6 \%$ & 22 & $12.2 \%$ & 49 & $8.8 \%$ \\
\hline Meningitis & 4 & $0.5 \%$ & 1 & $0.6 \%$ & 3 & $0.5 \%$ \\
\hline Spinal cord lesion & 5 & $0.7 \%$ & 1 & $0.6 \%$ & 4 & $0.7 \%$ \\
\hline Stroke & 51 & $6.9 \%$ & 13 & $7.2 \%$ & 38 & $6.8 \%$ \\
\hline Cranial nerve palsy & 4 & $0.5 \%$ & 1 & $0.6 \%$ & 3 & $0.5 \%$ \\
\hline Sensory peripheral neuropathy & 56 & $7.6 \%$ & 22 & $12.2 \%$ & 34 & $6.1 \%$ \\
\hline Motor mononeuritis multiplex & 4 & $0.5 \%$ & 1 & $0.6 \%$ & 3 & $0.5 \%$ \\
\hline
\end{tabular}


Table 2 continued

\begin{tabular}{llll}
\hline & $\begin{array}{l}\text { All MPA patients } \\
(N=740)\end{array}$ & $\begin{array}{l}\text { MPA patients with } \\
\text { relapse }(N=180)\end{array}$ & $\begin{array}{l}\text { MPA patients without } \\
\text { relapse }(N=560)\end{array}$ \\
\hline Relapse & & & \\
Presence of any relapse, $N(\%)$ & 180 & $24.3 \%$ & \\
Presence of a renal relapse, $N(\%)$ & 118 & $15.9 \%$ \\
Presence of a pulmonary relapse, $N(\%)$ & 6 & $0.8 \%$ \\
Presence of another relapse**, $N(\%)$ & 17 & $2.3 \%$ \\
Presence of a utilization relapse, $N(\%)$ & 39 & $5.3 \%$ & \\
Presence of a mixed relapse, $N(\%)$ & 13 & $1.8 \%$ & \\
\hline
\end{tabular}

$B V A S$ Birmingham Vasculitis Activity Scale, $C C I$ Charlson Comorbidity Index, $M P A$ microscopic polyangiitis, $S D$ standard deviation

${ }^{*}$ Patients with hemoptysis (ICD-9 CM 786.30, 786.39) were captured because no diagnosis codes were available for alveolar hemorrhage

** Other relapse-associated condition

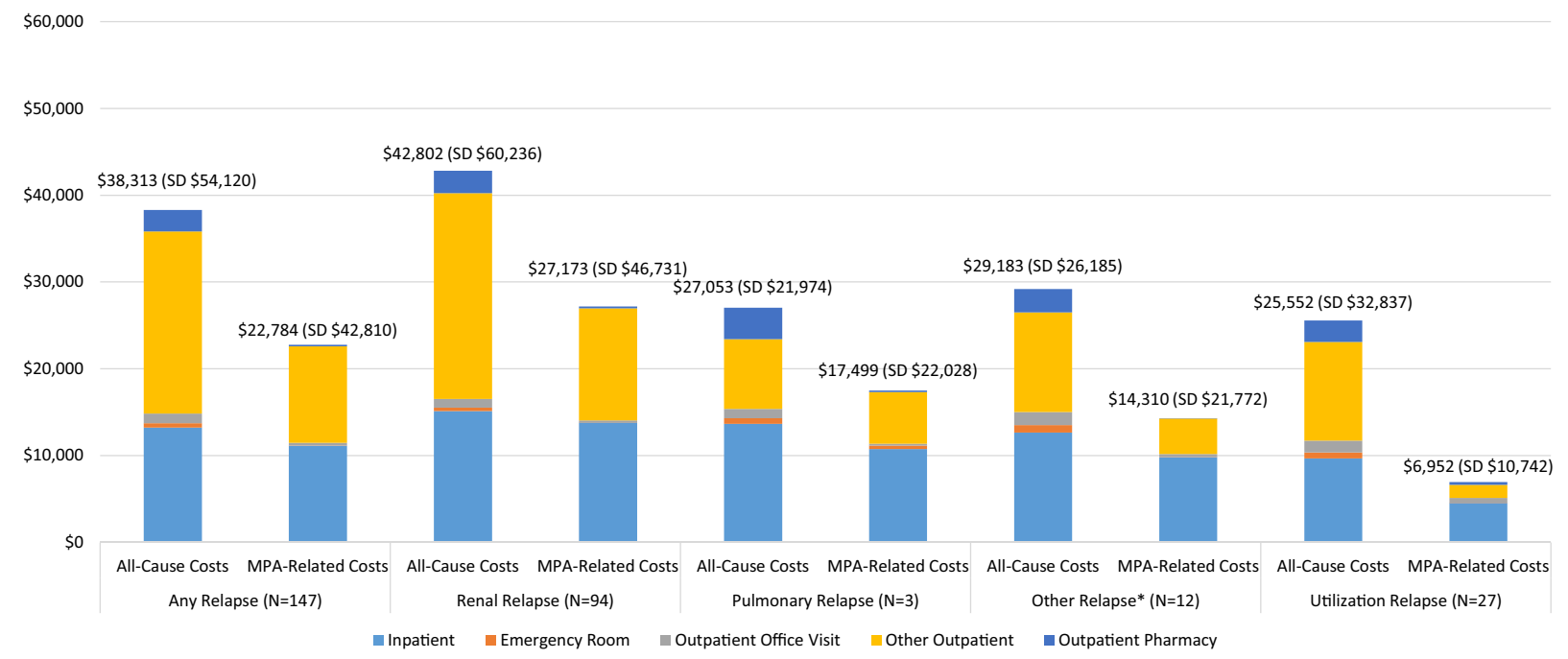

Fig. 2 Healthcare costs in the 4 months following first relapse, stratified by relapse type, for MPA patients. Note: costs were assessed only among patients with 4 months of continuous enrollment following first relapse. The costs

costs of 12 months' follow-up for patients with GPA who did not experience a relapse. Effective treatment that prevents relapses in this population appears to be of key importance in reducing the costs of care in GPA. following mixed relapses were included in costs after any relapse but were not reported separately. $M P A$ microscopic polyangiitis. Asterisk other relapse associated condition

Several analyses have found that relapses are common among patients with ANCA-associated vasculitis, especially with GPA. Studies have reported that over $\approx 4$ years of follow-up, $35-54 \%$ of patients with GPA experience a relapse [6-8]. Relapses typically require 
treatment, which can be very costly. To our knowledge, ours is the first analysis to quantify the healthcare costs incurred following a relapse. A prior claims-based analysis of patients with GPA found that, over a 12-month period, patients with GPA who were treated for a relapse had substantially higher all-cause healthcare costs than patients with GPA who did not experience a relapse (mean, $\$ 88,065$ vs. $\$ 30,682$, respectively) [10]. This prior analysis did not focus on costs incurred immediately after a relapse and did not attempt to classify types of relapses by organ system. Additionally, costs in the prior study were measured over 12 months rather than a 4-month period, resulting in higher costs.

This analysis found that renal relapses resulted in greater healthcare costs than other types of relapses. Renal involvement is very common in patients with GPA and MPA and can lead to severe consequences [14]. It can present as hematuria, proteinuria, renal failure, or nephritis [14]. Even though, with prompt and adequate therapy, patients often recover their renal function to the extent allowing for discontinuation of renal dialysis, end-stage renal failure can be the result of multiple renal relapses resulting in additive and, ultimately, irreversible renal damage $[14,15]$. End-stage renal failure is associated with a greater risk of death among patients with ANCA-associated vasculitis [16, 17]. For patients who fail to respond to immunosuppressive medications, dialysis must often be used. While in some individuals the renal function may eventually recover, others become chronically dialysis dependent [18-20] or require renal transplant. While we measured healthcare costs within 4 months of a renal relapse, permanent renal dysfunction and dialysis can occur [15, 21], which increases long-term healthcare costs [22]. Additionally, dialysis is associated with several undesirable outcomes, including depression, metabolic abnormalities, and chronic pain (among others), all of which negatively affect patients' quality of life [23] and increase the economic burden [22]. Some rheumatologists feel that sequential monitoring of ANCA titers can be used to monitor disease and prevent renal relapses [24]. However, monitoring is not universally accepted [14] and this study did not evaluate the costs of monitoring relative to relapses.

This analysis has limitations. First, administrative claims are generated for billing purposes, not research. Because of this, diagnoses may have been misclassified. Similarly, as no specific diagnosis code for MPA existed, an algorithm had to be applied to select these patients. This algorithm was not previously validated, and misclassification of MPA diagnosis might have occurred. Future research should validate this claims-based algorithm using other sources, such as electronic medical records. Additionally, in claims, inpatient medication use cannot be identified or disaggregated from the cost of the hospitalization. Second, clinical details on disease extent and severity, including BVAS scores, laboratory test values, and information about relapses and remissions, were not available. Therefore, a proxy for a relapse was developed using diagnosis codes for the major items on the BVAS scale in combination with medication use. The true proportion of patients with MPA with a relapse might have been overestimated, as renal and lung involvement was part of the algorithm used to identify patients. Additionally, while a GPA/unspecified arteritis diagnosis on an inpatient or emergency room claim and treatment consistent with a relapse were required, the clinical reason for a utilization relapse may have been something other than GPA or MPA. Third, the length of a relapse could not be determined from claims; thus, the costs were assessed over a 4-month time window based on the average length of relapse previously used in a clinical trial [12]. An individual's relapse might have lasted shorter or longer than 4 months. Fourth, we also required that all patients had 12 months of continuous enrollment following the index date and, specifically, 4 months of continuous enrollment following a relapse. This requirement was applied so that relapses and their costs could be measured over specific time periods for all patients; however, patients who died would have been excluded. These patients might have been more vulnerable to relapses, which might have resulted in higher costs due to treatment. Thus, in the current study, the true costs 
associated with relapse might have been underestimated. Fifth, some types of relapses, such as pulmonary relapses, were uncommon in this sample, therefore the cost estimates might have been biased by outliers. Sixth, no statistical analysis was done to compare the costs in patients with a relapse with those in patients without a relapse. However, the costs in the 4 months following relapses were substantially higher than those expected over a 4-month period in patients without a relapse, based on 12-month follow-up costs in this analysis. Comparative analyses of patients with and without relapse and different types of relapses, controlling for confounding, are potential avenues for future research, as is the comparison of costs of remission maintenance strategies with the costs of relapse. Finally, the results of this analysis may not be generalizable to other populations, including those with traditional Medicare (not Medicare supplemental) or Medicaid coverage or the uninsured.

\section{CONCLUSIONS}

Relapses are common in patients with GPA and MPA and can significantly impact the costs of treatment in GPA and MPA. In particular, the costs of treating relapses with renal involvement are very high as, among other reasons, the treatment often necessitates the use of dialysis. Further development of effective therapies for GPA and MPA is needed to offer effective, relapse-preventing therapies to the patients, an important goal from both the clinical and economic perspectives.

\section{ACKNOWLEDGEMENTS}

Funding. This study was funded by Genentech, Inc. Article processing charges were funded by Genentech, Inc. Truven Health Analytics received funds to conduct analyses and to draft the manuscript; however, authors did not directly receive funding for writing or co- authoring. All authors had full access to all of the data in this study and take complete responsibility for the integrity of the data and accuracy of the data analysis.

Authorship. All named authors meet the International Committee of Medical Journal Editors (ICMJE) criteria for authorship for this manuscript, take responsibility for the integrity of the work as a whole, and have given final approval to the version to be published. The authors acknowledge input on the study design by Karina Raimundo, of Genentech, Inc.

Disclosures. AM Kong is an employee of Truven Health Analytics. G Kim is an employee of Truven Health Analytics. M Michalska is an employee and stockholder of Genentech, Inc. $\mathrm{JH}$ Best is an employee and stockholders of Genentech, Inc.

Compliance with Ethics Guidelines. This article is based on previously conducted data, and does not involve any new studies of human or animal subjects performed by any of the authors.

Data Availability. The data used for this analysis were from proprietary databases and could not be made publicly available due to agreements between Truven Health Analytics and the data contributors. More information about the data can be obtained by contacting the corresponding author or by visiting http:// truvenhealth.com/markets/life-sciences/produ cts/data-tools/marketscan-databases.

Open Access. This article is distributed under the terms of the Creative Commons Attribution-NonCommercial 4.0 International License (http://creativecommons.org/licenses/ by-nc/4.0/), which permits any noncommercial use, distribution, and reproduction in any medium, provided you give appropriate credit to the original author(s) and the source, provide a link to the Creative Commons license, and indicate if changes were made. 


\section{REFERENCES}

1. Jennette JC, Falk RJ, Bacon PA, et al. 2012 revised International Chapel Hill Consensus Conference nomenclature of vasculitides. Arthritis Rheum. 2013;65:1-11.

2. Ntatsaki E, Carruthers D, Chakravarty K, et al. BSR and BHPR guideline for the management of adults with ANCA-associated vasculitis. Rheumatology (Oxford). 2014;53:2306-9.

3. Tarzi RM, Pusey CD. Current and future prospects in the management of granulomatosis with polyangiitis (Wegener's granulomatosis). Ther Clin Risk Manag. 2014;2014:279-93.

4. Bosch X, Guilabert A, Espinosa G, Mirapeix E. Treatment of antineutrophil cytoplasmic antibodyassociated vasculitis. JAMA. 2007;298:655-69.

5. Koldingsnes W, Nossent JC. Baseline features and initial treatment as predictors of remission and relapse in Wegener's granulomatosis. J Rheumatol. 2003;30:80-8.

6. Hogan SL, Falk RJ, Chin $\mathrm{H}$, et al. Predictors of relapse and treatment resistance in antineutrophil cytoplasmic antibody-associated small-vessel vasculitis. Ann Intern Med. 2005;143:621-31.

7. Pagnoux C, Hogan SL, Chin H, et al. Predictors of treatment resistance and relapse in antineutrophil cytoplasmic antibody-associated small-vessel vasculitis: comparison of two independent cohorts. Arthritis Rheum. 2008;58:2908-18.

8. Holle JU, Gross WL, Latza U, et al. Improved outcome in 445 patients with Wegener's granulomatosis in a German vasculitis center over four decades. Arthritis Rheum. 2011;63:257-66.

9. Stone JH, Hoffman GS, Merkel PA, et al. A diseasespecific activity index for Wegener's granulomatosis. Arthritis Rheum. 2001;44:912-20.

10. Raimundo K, Farr AM, Kim G, Duna G. Clinical and economic burden of antineutrophil cytoplasmic antibody-associated vasculitis in the United States. J Rheumatol. 2015;42:2383-91.

11. Truven Health Analytics. MarketScan studies: abbreviated bibliography. [Internet. Accessed 21 June 2017]. http://sites.truvenhealth.com/bibliography/ 2014TruvenHealthMarketScanBibliography.pdf.

12. Stone JH, Merkel PA, Spiera R, et al. Rituximab versus cyclophosphamide for ANCA-associated vasculitis. N Engl J Med. 2010;363:221-32.
13. Deyo RA, Cherkin DC, Ciol MA. Adapting a clinical comorbidity index for use with ICD-9-CM administrative databases. J Clin Epidemiol. 1992;45:613-9.

14. Rowaiye OO, Kusztal M, Klinger M. The kidneys and ANCA-associated vasculitis: from pathogenesis to diagnosis. Clin Kidney J. 2015;8:343-50.

15. Moiseev S, Novikov P, Jayne D, Mukhin N. Endstage renal disease in ANCA-associated vasculitis. Nephrol Dial Transplant. 2017;32:248-53.

16. Little MA, Pusey CD. Glomerulonephritis due to antineutrophil cytoplasm antibody-associated vasculitis: an update on approached to management. Nephrology (Carlton). 2005;10:368-76.

17. Booth $\mathrm{AD}$, Almond MK, Burns A, et al. Outcome of ANCA-associated renal vasculitis: a 5-year retrospective study. Am J Kidney Dis. 2003;41:776-84.

18. Mekhail TM, Hoffman GS. Long-term outcome of Wegener's granulomatosis in patients with renal disease requiring dialysis. J Rheumatol. 2000;27:1237-40.

19. Lee T, Gasim A, Derebail VK, et al. Predictors of treatment outcomes in ANCA-associated vasculitis with severe kidney failure. Clin J Am Soc Nephrol. 2014;9:905-13.

20. Manno RL, Seo P, Geetha D. Older patients with ANCA-associated vasculitis and dialysis dependent renal failure: a retrospective study. BMC Nephrol. 2015; $16: 88$.

21. Slot MC, Tervaert JW, Franssen CF, Stegeman CA. Renal survival and prognostic factors in patients with PR3-ANCA associated vasculitis with renal involvement. Kidney Int. 2003;63:670-7.

22. Saran R, Li Y, Robinson B, et al. US Renal Data System 2015 annual data report: epidemiology of kidney disease in the United States. Am J Kidney Dis. 2016;67(3 Suppl 1):S1-305.

23. Weisbord SD. Patient-centered dialysis care: depression, pain, and quality of life. Semin Dial. 2016;29:158-64.

24. Kemna MJ, Damoiseaux J, et al. ANCA as a predictor of relapse: useful in patients with renal involvement but not in patients with nonrenal disease. J Am Soc Nephrol. 2015;26:537-42. 